Pacific Journal of Mathematics

EMBEDDING THEOREMS FOR COMMUTATIVE BANACH 


\title{
EMBEDDING THEOREMS FOR COMMUTATIVE BANACH ALGEBRAS
}

\author{
William G. Bade and Philip C. Curtis, Jr.
}

One knows from the Gelfand theory that every commutative semisimple Banach algebra $\mathfrak{U}$ containing an identity is a separating subalgebra of the algebra of all complex continuous functions on the space of maximal ideals of $\mathfrak{A}$. We shall be concerned in this paper with conditions which when imposed on a separating Banach subalgebra $\mathfrak{U}$ of $C(\Omega), \Omega$ a compact Hausdorff space, will guarantee that $\mathfrak{X}=C(\Omega)$. The conditions will take the form of restrictions on either the algebra or the space $\Omega$. For example we prove that if $\mathscr{U}$ is an $\varepsilon$-normal Banach subalgebra of $C(\Omega)$ then $\mathfrak{U}=C(\Omega)$ if an appropriate boundedness condition holds locally on $\Omega$. If $\Omega$ is assumed to be an $F$ space in the sense of Gillman and Henriksen this boundedness assumption is redundant. These results include a recent characterization of Sidon sets in discrete groups due to Rudin and have applications to interpolation problems for bounded analytic functions.

Various conditions which guarantee that $\mathfrak{A}=C(\Omega)$ are known. One, due to Glicksberg [5], is the following.

(1) Assume $\mathfrak{A}$ is sup-norm closed, contains the constants, and in addition assume that the restriction of $\mathfrak{A}$ to each closed subset $F$ of $\Omega$ is a closed subalgebra of $C(F)$.

Another, due to the present authors [1], is the following:

(2) Assume $\Omega$ is a totally disconnected $F$-space and that $\Omega$ is the maximal ideal space for $\mathfrak{A}$.

A compact space $\Omega$ is an $F$-space if disjoint open $F_{\sigma}$ sets in $\Omega$ have disjoint closures. This class of spaces was introduced by Gillman and Henriksen in [4] and includes stonian and $\sigma$-stonian spaces as well as their closed subsets. There are also connected examples.

- The results in this paper center around extensions of these conditions as well as others due to Katznelson [11, 12]. Many of the techniques apply equally well in a Banach space setting, and are discussed in this way where possible.

To begin the discussion we need the following definition: given $\varepsilon>0$, call a subset $\mathfrak{F} \subset C(\Omega)$ an $\varepsilon$-normal family if for each pair $F_{1}, F_{2}$ of disjoint compact subsets of $\Omega$ there exists an $x \in \widetilde{F}$ satisfying
(i ) $|x(\omega)-1|<\varepsilon$, $\omega \in F_{1}$,
(ii) $|x(\omega)|<\varepsilon$,
$\omega \in F_{2}$.

By a Banach subalgebra of $C(\Omega)$ we will mean a subalgebra of $C(\Omega)$, not necessarily containing the constants, which is a Banach algebra 
under some norm.

The result of the paper which illustrates the unusual properties of $F$-spaces is the following:

THeorem A. Let $\Omega$ be a compact $F$-space and $\mathfrak{U}$ be a Banach subalgebra of $C(\Omega)$. If $\mathfrak{A}$ is an $\varepsilon$-normal subalgebra for an $\varepsilon<1 / 2$ (in particular if $\mathfrak{A}$ is dense) then $\mathfrak{U}=C(\Omega)$.

In addition to extending the result of [1] mentioned in (2) above, this theorem contains Rudin's characterization of Sidon sets in discrete groups and has applications to interpolation problems for bounded analytic functions.

For general compact spaces $\Omega$, we will call an $\varepsilon$-normal family contained in a Banach subalgebra of $C(\Omega)$, or more generally contained in the continuous image in $C(\Omega)$ of a Banach space $X$, locally bounded if for each point $\omega$ there exists a compact neighborhood $N_{\omega}$ such that whenever the sets $F_{1}, F_{2}$ belong to $N_{\omega}$, the $x$ may be chosen to have $\mathfrak{U}$-norm (or $X$ norm) less than a constant depending on $\omega$.

Our extension of (1) and the results of Katznelson is basically contained in the following:

Theorem B. Let $\Omega$ be a compact Hausdorff space, $\mathfrak{X}$ a Banach subalgebra of $C(\Omega)$. Then $\mathfrak{A}=C(\Omega)$ if the following conditions are satisfied.

(1) $\mathfrak{A}$ is an $\varepsilon$-normal family for some $\varepsilon<1 / 2$.

(2) $\mathfrak{X}$ is locally bounded.

If $\Omega$ is an $F$-space (1) implies (2), thus proving Theorem A. Indeed for $F$-spaces much can be said when $\mathfrak{A}$ is only assumed to be the continuous image of a Banach space $X$.

Theorem C. Let $\Omega$ be a compact $F$-space and $T$ a continuous linear map of a Banach space $X$ into $C(\Omega)$ such that $T X$ forms an $\varepsilon$-normal family for some $\varepsilon<1 / 4$. Then there exists a finite open covering $U_{1}, \cdots, U_{n}$ of $\Omega$ such that

$$
T X \mid \bar{U}_{i}=C\left(\bar{U}_{i}\right), i=1, \cdots, n .
$$

In general, linear subspaces of $C(\Omega)$ may be $\varepsilon$-normal, $\varepsilon<1 / 4$, without being dense, but for totally disconnected spaces density and $\varepsilon$-normality are equivalent. A short argument shows that $\varepsilon$-normality and density are also equivalent for arbitrary $F$-spaces.

Theorem $C$ raises the question whether a continuous linear map $T$ of a Banach space $X$ into $C(\Omega), \Omega$ an $F$-space, which has dense 
range, must be onto $C(\Omega)$. Section 4 contains an example due to J. Lindenstrauss which shows that $T X \neq C(\Omega)$ in general. G. Seever [16] has proved that $T X=C(\Omega)$ if one has the stronger assumption that $T X$ is normal on $\Omega$. We give a new proof of Seever's theorem. An unpublished result of Beurling which covers the case when $X$ is an adjoint space, $C(\Omega)=l_{\infty}$, and $T$ is weak star continuous is proved by an argument of Helson.

We are indebted to J. Lindenstrauss and Y. Katznelson for the elegant examples in $\S 4$, and to Katznelson for illuminating discussions of the problems of this paper.

1. Preliminaries. In this section we collect some facts about approximation and onto maps. Given a bounded linear map $T: X \rightarrow C_{0}(\Omega)$, where $\Omega$ is locally compact and $X$ is a Banach space, one seeks conditions to insure that $T X=C_{0}(\Omega)$. These results are not new for the most part, and the techniques appear in a variety of settings. We first consider the general case $T: X \rightarrow Y$, where $Y$ is a Banach space. Recall that a subset $E \subseteq Y$ is equilibrated if $y$ in $E$ and $|\alpha| \leqq 1$ implies $\alpha y \in E$. The smallest convex equilibrated set containing $E$ is denoted by $\operatorname{coe}(E)$, the smallest convex set by $c o(E)$, their respective closures by $\overline{c o e}(E)$ and $\overline{c o}(E)$.

Lemma 1.1. Let $E$ be a subset of $Y$. Then $\overline{c o e}(E)$ contains the closed unit ball $S$ of $Y$ if and only if

$$
\left\|y^{*}\right\| \leqq \sup _{y \in E}\left|y^{*}(y)\right|
$$

for each $y^{*} \in Y^{*}$.

Proof. Note $\operatorname{coe}(E)$ consists of all sums $\sum_{i=1}^{n} \alpha_{i} y_{i}$, where $y_{i} \in E$ and $\sum_{i=1}^{n}\left|\alpha_{i}\right| \leqq 1$. If $\overline{c o e}(E) \supseteq S$, then for each $y^{*} \in Y^{*}$ and $\varepsilon>0$ we can find $y_{0}=\sum_{i=1}^{n} \alpha_{i} y_{i} \in \operatorname{coe}(E)$ such that

$$
\left|y^{*}\left(y_{0}\right)\right|>\| y^{*}||-\varepsilon .
$$

For some $i$ we must have $\left|y^{*}\left(y_{i}\right)\right|>\left\|y^{*}\right\|-\varepsilon$. Conversely, if $y_{0} \in S$, but $y_{0} \notin \overline{c o e}(E)$, the separation theorem [3, p. 417] yields a functional $y_{0}^{*}$ such that

$$
\left\|y_{0}^{*}\right\| \geqq \operatorname{Re} y_{0}^{*}\left(y_{0}\right)>\sup \left\{\operatorname{Re} y_{0}^{*}(y): y \in \overline{\operatorname{coe}}(E)\right\} .
$$

Since $\overline{c o e}(E)$ is equilibrated, we have

$$
\left\|y_{0}^{*}\right\|>\sup \left|y_{0}^{*}(y)\right| \text {. }
$$

Theorem 1.2. Let $T: X \rightarrow Y$ be linear and continuous. Suppose 
there exists a set $E \subseteq Y$ and constants $k<1$ and $K$ such that

(1) $\overline{c o e}(E)$ contains the closed unit ball $S$ of $Y$.

(2) For each $y \in E$ there exists an $x \in X$ such that

$$
\|T x-y\| \leqq k,\|x\| \leqq K .
$$

Then $T X=Y$. If $T$ is one to one, $\left\|T^{-1}\right\| \leqq K(1-k)^{-1}$.

Proof. For any $y^{*} \in Y^{*}$ and $y \in E$, select $x$ by (2) and note

$$
\begin{aligned}
\left|y^{*}(y)\right| & \leqq\left|y^{*}(y-T x)\right|+\left|y^{*}(T x)\right| \\
& \leqq k\left\|y^{*}\right\|+\left|\left(T^{*} y^{*}\right)(x)\right| \\
& \leqq k\left\|y^{*}\right\|+K\left\|T^{*} y^{*}\right\| .
\end{aligned}
$$

Taking sups on $y$ in $E$ yields

$$
\left\|y^{*}\right\| \leqq k\left\|y^{*}\right\|+K\left\|T^{*} y^{*}\right\|
$$

by Lemma 1. Thus

$$
\left\|T^{*} y^{*}\right\| \geqq K^{-1}(1-k)\left\|y^{*}\right\|, y^{*} \in Y^{*},
$$

showing $T^{*}$, and hence $T$ has a closed range [3, p. 488]. However the argument above shows $T X$ is dense in $Y$, since if $y^{*}(T X)=0$, $y^{*} \neq 0$, then

$$
\left|y^{*}(y)\right| \leqq k\left\|y^{*}\right\|<\left\|y^{*}\right\|, y \in E,
$$

in contradiction of Lemma 1.1. The result now follows.

Theorem 1.2 is due to Katznelson [11] in slightly different form. He constructs a solution of $T x=y$ by successive approximations. The proof above follows an argument of Rudin to prove a theorem on Helson sets [15, p. 116], (Corollary 1.3 below) which is a special case of Theorem 1.1. Recall that if $G$ is a locally compact abelian group, a compact set $P \subseteq G$ is a Helson set if each continuous function on $P$ is the restriction $\hat{f} \mid P$ of the Fourier transform of an element of $L_{1}$ on the dual group $\Gamma$.

Corollary 1.3. Suppose $P$ is compact in $G$ and there exist constants $k<1$ and $K$ such that to each $F \in C(P)$ with $|F(t)|=1$, $t \in P$, there corresponds an element $f \in L_{1}(\Gamma)$ such that $\|f\|_{1} \leqq K$ and

$$
\sup _{t \in P}|\hat{f}(t)-F(t)| \leqq k \text {. }
$$

Then $P$ is a Helson set.

Proof. Define $T: L_{1}(\Gamma) \rightarrow C(P)$ by $T f=\hat{f} \mid P$. The extreme points of the unit ball $S$ in $C(P)$ are precisely the functions of absolute value 
one. Lemma 1.1 and Lemma 5.5.1 of [15] show $S$ is the norm closed convex hull of its set of extreme points. The result now follows directly from Theorem 1.2.

We next consider the case that $Y$ is $C_{0}(\Omega)$, the continuous functions vanishing at infinity on a locally compact Hausdorff space. A subset $E$ of $C_{0}(\Omega)$ is a normal family if for each pair $F_{1}$, and $F_{2}$ of disjoint compact sets in $Q$ there exists $f \in E$ with $f\left(F_{1}\right)=0, f\left(F_{2}\right)=1$. We denote the nonnegative functions in the closed unit ball $S$ of $C_{0}(\Omega)$ by $S_{+}$.

Lemma 1.4. If $E$ is a bounded normal family, then $\overline{c o}(E)$ contains $S_{+}$.

Proof. It suffices to approximate any element $f$ of $S_{+}$having compact support by elements of $c o(E)$. Let $n$ be any positive integer and $L=\sup \{\|f\|: f \in E\}$. Let $C$ be a compact set whose interior contains the support of $f$. Define

$$
\begin{aligned}
& U_{k}=\left\{\omega \in \Omega: f(\omega) \geqq \frac{k}{n}\right\}, \\
& V_{k}=\left\{\omega \in C: f(\omega) \leqq \frac{k-1}{n}\right\}, \quad k=1,2, \cdots, n-1 .
\end{aligned}
$$

Choose $f_{1} \in E$ with

$$
f_{1}\left(U_{1}\right)=1, \quad f_{1}\left(V_{1}\right)=0 .
$$

Let $W_{1}=\left\{\omega \notin C:\left|f_{1}(\omega)\right| \geqq 1 / n^{2}\right\}$. Then $W_{1}$ is compact. Choose $f_{2} \in E$ with

$$
f_{2}\left(U_{2}\right)=1, \quad f_{2}\left(V_{2} \cup W_{1}\right)=0 .
$$

Let $W_{2}=\left\{\omega \notin C:\left|f_{2}(\omega)\right| \geqq 1 / n^{2}\right\}$, etc. Continuing, we finally obtain $f_{n-1} \in E$ with

$$
\begin{aligned}
f_{n-1}(\omega) & =1, \quad \omega \in U_{n-1} \\
& =0, \quad \omega \in V_{n-1} \cup W_{1} \cup \cdots \cup W_{n-2} .
\end{aligned}
$$

Define the function $g=1 / n \sum_{k=1}^{n-1} f_{k}$. We estimate $\|f-g\|_{\infty}$. Note that

$$
|f(\omega)-g(\omega)| \leqq \frac{L+1}{n}, \quad \omega \in C .
$$

Now $f(\omega)=0, \omega \notin C$, the sets $W_{i}$ are disjoint and compact, and

$$
|g(\omega)| \leqq \frac{1}{n^{2}}, \quad \omega \notin \bigcup_{i=1}^{n-2} W_{i} .
$$


If $\omega \in W_{i}$,

$$
\begin{aligned}
f_{j}(\omega) & =0, \quad j>i \\
\left|f_{j}(\omega)\right| & \leqq \frac{1}{n^{2}}, \quad j<i \\
\left|f_{i}(\omega)\right| & \leqq L .
\end{aligned}
$$

Thus

$$
|g(\omega)| \leqq \frac{1}{n} \sum_{j=1}^{i}\left|f_{j}(\omega)\right| \leqq \frac{1}{n^{2}}+\frac{L}{n}, \quad \omega \in W_{i}
$$

and we have proved

$$
\|f-g\|_{\infty} \leqq \frac{L+1}{n} .
$$

Now $h=n(n-1)^{-1} g \in \operatorname{co}(E)$. Since $n$ is arbitrary, the result follows.

TheOREM 1.5. Let $\Omega$ be locally compact and $T: X \rightarrow C_{0}(\Omega)$ be linear and continuous. Suppose there exist constants $\varepsilon<1 / 4$ and $M$ such that if $F_{1}$ and $F_{2}$ are any disjoint compact sets in $\Omega$, these exists an $x \in X$ such that

(i) $|(T x)(\omega)| \leqq \varepsilon, \quad \omega \in F_{1}$

(ii) $|(T x)(\omega)-1| \leqq \varepsilon, \quad \omega \in F_{2}$

(iii) $\|x\| \leqq M$.

Then $T X=C_{0}(\Omega)$. If $T$ is one-to-one then $\left\|T^{-1}\right\| \leqq 4 M(1-4 \varepsilon)^{-1}$.

Proof. Let $\varepsilon<\varepsilon^{\prime}<1 / 4$ and $E$ be any bounded normal family in $C_{0}(\Omega)$. If $F_{1}$ and $F_{2}$ are any disjoint compact sets, we can find $x \in X$ with

$$
\begin{gathered}
\|x\| \leqq M,\left|(T x)\left(F_{1}\right)-1\right| \leqq \varepsilon,\left|(T x)\left(F_{2}\right)\right| \leqq \varepsilon, \text { and } f \in E \text { with } \\
f\left(F_{1}\right)=1, \quad f\left(F_{2}\right)=0 .
\end{gathered}
$$

Since $C\left(F_{1} \cup F_{2}\right)$ is isometrically isomorphic with the quotient of $C_{0}(\Omega)$ by the ideal of functions zero on $F_{1} \cup F_{2}$, we can select $g_{f} \in C_{0}(\Omega)$ with $g_{f}\left(F_{1} \cup F_{2}\right)=0$ and

$$
\left\|T x-\left(f+g_{f}\right)\right\| \leqq \varepsilon^{\prime} .
$$

Then $E^{\prime}=\left\{f+g_{f}: f \in E\right\}$ is a bounded normal family, $\overline{c o}\left(E^{\prime}\right) \supseteq S_{+}$by Lemma 1.4, and hence $\overline{c o e}\left(4 E^{\prime}\right) \supseteq S$. For each $g \in 4 E^{\prime}$ we can find an $x$ with

$$
\|T x-g\| \leqq 4 \varepsilon^{\prime}, \quad\|x\| \leqq 4 M .
$$

Theorem 1.5 now follows from Theorem 1.2. 
REMARK. Often in applications it is just as convenient to verify that for each compact set $F \subseteq \Omega$ and $f \in C(F)$ there exists $x \in X$ satisfying $\|x\| \leqq M$ and $\|T x-f\|_{\infty} \leqq \varepsilon\|f\|_{\infty}$. This of course implies the condition of Theorem 1.5.

If $T X$ satisfies (i, ii) we shall call $T X$ an $\varepsilon$-normal family. If (iii) is satisfied we call $T X$ boundedly $\varepsilon$-normal. If $T X$ is $\varepsilon$-normal and for each $\omega \in \Omega$ (iii) holds when $F_{1}, F_{2}$ belong to a suitably small compact neighborhood of $\omega$, then we call $T X$ locally bounded. Note that Theorem 1.5 yields:

Corollary 1.6. If $\Omega$ is compact and $T X$ is e-normal and locally bounded, there exist finitely many compact sets $S_{1}, \cdots, S_{n}$ whose interiors cover $\Omega$ and such that $T X \mid S_{i}=C\left(S_{i}\right)$.

CoROLLARY 1.7. If $\Omega$ is compact and totally disconnected and $\mathfrak{M}$ is a linear subspace of $C(\Omega)$ which is $\varepsilon$-normal for $\varepsilon<1 / 4$, then $\mathfrak{M}$ is dense in $C(\Omega)$.

Proof. Let $X$ be the closure of $\mathfrak{M}$ in the sup-norm and $T$ be the natural injection map of $X$ into $C(\Omega)$. Since $F_{1}$ and $F_{2}$ may be enclosed in disjoint open and closed sets having $\Omega$ as their union condition (iii) of Theorem 1.5 is satisfied with $M=1+\varepsilon$.

It is important to note in the absence of boundedness 1.4 and 1.5 are false. The recent example of McKissick [13] of a sup-norm closed normal function algebra which is not $C(\Omega)$ provides a counter example.

We note in passing that if $\Omega$ is compact and $\omega \in \Omega$, then $T X=C(\Omega)$ if and only if $(T X)_{\omega}=C_{0}(\Omega \sim\{\omega\})$, where for a linear subspace $\mathfrak{M} \subseteq C(\Omega), \mathfrak{M}_{\omega}=\{f \in C(\Omega): f(\omega)=0\}$. Also, as shown by Seever [16], $T X=C(\Omega)$ if and only if for each measure $\mu$ on $\Omega$, the restriction of $T X$ to the carrier of $\mu$ is all continuous function on the carrier.

2. We now specialize to the situation when $X$ is a Banach algebra of continuous functions vanishing at $\infty$ on $\Omega$. In this case we shall write $\mathfrak{A}$ for $X$. Then of course $|x(\omega)| \leqq\|x\|$ for $x \in \mathfrak{A}$. Hence $\|x\|_{\infty} \leqq\|x\|$ and the embedding of $\mathfrak{A}$ into $C_{0}(\Omega)$ is continuous. Also if an algebra is $\varepsilon$-normal for some $\varepsilon<1 / 2$, it is $\varepsilon$-normal for every such $\varepsilon$. This is clear since the function $f$ defined by

$$
\begin{aligned}
f(z) & =0, \quad|z| \leqq \varepsilon \\
& =1, \quad|1-z| \leqq \varepsilon
\end{aligned}
$$

can be uniformly approximated on these sets by polynomials without constant term. Indeed, a condition equivalent to $\varepsilon$-normality for algebras is the following: For each pair of disjoint compact sets $S_{0}, S_{1}$ 
of $\Omega$, there exists an $x \in \mathfrak{A}$ and disjoint open sets $V_{0}, V_{1}$ of complex numbers such that $x\left(S_{1}\right) \subset V_{i}$, and $C \sim\left(\overline{V_{0} \cup V_{1}}\right)$ is connected and contains the origin.

That this is equivalent to $\varepsilon$-normality is easily seen. If $\varepsilon<1 / 6$ and $\left|x\left(S_{0}\right)\right| \leqq \varepsilon,\left|1-x\left(S_{1}\right)\right| \leqq \varepsilon,\left|1-y\left(S_{0}\right)\right| \leqq \varepsilon$, and $\left|y\left(S_{1}\right)\right| \leqq \varepsilon$, then $2 x+y$ has the desired property. For the converse note that without loss of generality we may assume that $\bar{V}_{0} \cap \bar{V}_{1}=\varnothing$. Let $W$ be an open neighborhood of 0 satisfying $W \subset \bar{W} \subset C \sim\left(\overline{V_{0} \cup V_{1}}\right)$ and such that $C \sim\left(\overline{V_{0} \cup V_{1} \cup W}\right)$ is connected. Then by the theorem of Mergelyan, the function $f$ defined by $f\left(V_{0} \cup W\right)=0, f\left(V_{1}\right)=1$ may be uniformly approximated on $\overline{V_{0} \cup V_{1} \cup W}$ by polynomials $p(z)$. Since these polynomials may be taken to have no constant term, if $x\left(S_{i}\right) \subset V_{i}$, $i=1,2$; then $p_{n}(x) \in \mathfrak{A},\left|p_{n}(x)\left(S_{0}\right)\right|<\varepsilon$, and $\left|1-p_{n}(x)\left(S_{1}\right)\right|<\varepsilon$.

Suppose now $\Omega$ is compact and the $\varepsilon$-normal Banach subalgebra of $C(\Omega)$ is locally bounded. Then by Corollary 1.6 there exist finitely many compact sets $S_{1}, \cdots, S_{n}$ whose interiors cover $\Omega$ such that $\mathfrak{U} \mid S_{i}=C\left(S_{i}\right)$. We shall prove $\mathfrak{A}=C(\Omega)$. Incidentally the ability to match each continuous function on the sets of a covering is not sufficient to prove $\mathfrak{A}=C(\Omega)$ without the assumption of $\varepsilon$-normality. To see this let

$$
\mathfrak{U}=\left\{x \in l_{\infty}: x(n)=x(-n)\right\} .
$$

For $\mathfrak{A}$ restricted to the negative or to the nonnegative integers yields all bounded sequences on these sets, but $\mathfrak{A} \neq l_{\infty}$. We begin with the following

THeOREM 2.1. Let $\Omega$ be a compact Hausdorff space and $\mathfrak{A}$ be an E-normal Banach subalgebra of $C(\Omega)$. Suppose there exists a finite covering of $\Omega$ by open sets $U_{i}$ such that $\mathfrak{A} \mid \bar{U}_{i}=C\left(\bar{U}_{i}\right)$. Then $\mathfrak{A}=C(\Omega)$.

Proof. We first make a definition.

Let $U_{1}, \cdots, U_{n}$ be an open covering of a topological space. An $\varepsilon$-partition of unity subordinate to this covering is a set of continuous functions $f_{1}, \cdots, f_{n}$ such that

$$
\begin{aligned}
& \left|f_{i}(\omega)\right| \leqq \varepsilon \quad \omega \notin U_{i} \\
& f_{1}+\cdots+f_{n}=1 .
\end{aligned}
$$

Lemma 2.2. Let $\mathfrak{A}$ satisfy the hypothesis of Theorem 2.1. Then for each $\varepsilon>0 \mathfrak{U}$ contains an $\varepsilon$-partition of unity subordinate to the given covering.

Proof. We observe first the following identity for complex numbers $\lambda_{1}, \cdots, \lambda_{n}$ 


$$
\text { (\#) } \prod_{i=1}^{n}\left(1-\lambda_{i}\right)=1-\lambda_{1} \prod_{i=2}^{n}\left(1-\lambda_{i}\right)-\lambda_{2} \prod_{i=3}^{n}\left(1-\lambda_{i}\right)-\cdots-\lambda_{n}
$$

which is easily proved by induction. Next we assert that if $U$ is one of the open sets $U_{i}$, then there is a constant $M$ such that for each compact set $S \subseteq U$ and $\varepsilon>0$ there exists $x \in \mathfrak{A}$ satisfying $|x(\omega)| \leqq \varepsilon$ off $U, x(S)=1$ and $\|x\|_{\infty} \leqq M$. To prove this, note that by the closed graph theorem there is a constant $C$ such that if $f \in C(\bar{U})$, then $\hat{f}$ may be chosen in $\mathfrak{A}$ satisfying

$$
\begin{aligned}
\hat{f} & =f \text { on } \bar{U}, \quad \text { and } \\
\|\hat{f}\| & \leqq C\|f\|_{\infty} .
\end{aligned}
$$

Let $0<\varepsilon<1 / 2$. Pick $h \in \mathfrak{U}$ satisfying

$$
\begin{aligned}
|1-h(\omega)| & \leqq \varepsilon & & \omega \in S \\
|h(\omega)| & \leqq \varepsilon & & \omega \notin U .
\end{aligned}
$$

Let $T=\{\omega:|h(\omega)| \geqq 1 / 2\}$. By the Tietze extension theorem there exists $g \in C(\bar{U})$ satisfying $g(\omega)=h^{-1}(\omega), \omega \in T$,

$$
\|g\|_{\infty} \leqq 2 \text {. }
$$

Therefore if $\hat{g} \in \mathfrak{A}, \hat{g}=g$ on $U$, and $\|\hat{g}\| \leqq 2 C$, we may take $x=\hat{g} h$. Then $|x(\Omega \sim U)| \leqq 2 C \varepsilon, x(T)=1$, and $\|x\|_{\infty} \leqq C$.

Now select open sets $V_{i}, i=1, \cdots, n$, covering $\Omega$ and satisfying $V_{i} \subset \bar{V}_{i} \subset U_{i}$. Choose $f_{n}, \cdots, f_{1} \in \mathfrak{X}$ in turn such that

$$
\begin{aligned}
\left|f_{k}(\omega)\right| & \leqq \varepsilon_{k} \text { off } U_{k} \\
f_{k}(\omega) & =1 \text { on } V_{k}
\end{aligned}
$$

where $\varepsilon_{n}=\varepsilon, \varepsilon_{k}=\varepsilon\left\|\prod_{k+1}^{n}\left(1-f_{i}\right)\right\|^{-1}, k=1, \cdots, n-1$. Let $x_{k}=$ $f_{k} \prod_{k+1}^{n}\left(1-f_{i}\right) ; x_{k} \in \mathfrak{U}$ and $\left|x_{k}(\omega)\right| \leqq \varepsilon$ off $U_{k}$. Since $\prod_{k=1}^{n}\left(1-f_{k}\right)(\omega)=$ $=0, \omega \in \Omega$, it follows from (\#) that $1=x_{1}+\cdots+x_{n}$.

To finish the proof of the theorem we apply 1.2. That is, we assert for $0<\varepsilon<1$ there is a constant $M$ such that for $f \in C(\Omega)$, $\|f\|_{\infty} \leqq 1$, we may choose $\hat{f} \in \mathfrak{A}$ satisfying

$$
\begin{aligned}
\|f-\hat{f}\| & \leqq \varepsilon \\
\|\hat{f}\| & \leqq M .
\end{aligned}
$$

To do this observe that there exists a constant $K$ and functions $f_{1}, \cdots, f_{n} \in \mathfrak{A}$ satisfying $f_{i}=f$ on $U_{i}$ and $\left\|f_{i}\right\| \leqq K$. Let $x_{1}, \cdots, x_{n}$ be the $\varepsilon$-partition constructed via Lemma 2.2. If $\hat{f}=f_{1} x_{1}+\cdots+f_{n} x_{n}$, then $\hat{f} \in \mathfrak{A}$. Also $\|\hat{f}\| \leqq K \sum_{i=1}^{n}\left\|x_{i}\right\|$ which we may take for $M$. To estimate $\|f-\hat{f}\|_{\infty}$ note that if $\omega \in U_{i}$, then $\left(f x_{i}-f_{i} x_{i}\right)(\omega)=0$. If $\omega \notin U_{i}$, then

$$
\left|\left(f x_{i}-f_{i} x_{i}\right)(\omega)\right| \leqq\left(\|f\|+\left\|f_{i}\right\|_{\infty}\right)\left|x_{i}(\omega)\right| \leqq(K+1) \varepsilon .
$$


Therefore

$$
\|f-\hat{f}\|_{\infty} \leqq \sum_{i=1}^{n}\left\|f x_{i}-f_{i} x_{i}\right\|_{\infty} \leqq n(K+1) \varepsilon<1
$$

for a suitable choice of $\varepsilon$. This finishes the proof.

CoRollary 2.3. Let $\mathfrak{H}$ be an e-normal Banach subalgebra of $C(\Omega)$, $\Omega$ compact Hausdorff. If $\mathfrak{U}$ is locally bounded then $\mathfrak{A}=C(\Omega)$.

We note that this corollary is a local version of the main theorem of [12]. For, Katznelson's condition that for each closed set $F$ of a compact space $\Omega$ there exists an $\varepsilon=\varepsilon(F)$ such that whenever $N$ is both closed and open in $F, \mathfrak{A}$ contains an element $h$ of norm one satisfying $\operatorname{Re}(x(\omega))<0, \omega \in N, \operatorname{Re}(x(\omega))>\varepsilon, \omega \in F \sim N$ is easily seen to be equivalent to 'bounded $\varepsilon$-normality'. That his condition implies the latter is implicit in Lemma 1 of [12], and the same sort of argument yields the converse.

Another related result is the theorem of Glicksberg [5] that if $\mathscr{O}$ is a closed separating subalgebra of $C(\Omega), \Omega$ compact, containing the constants such that the restriction to each closed subset $F \subset \Omega$ is a closed subalgebra of $C(F)$, then $\mathfrak{A}=C(\Omega)$. Theorem 2.2 yields a local version of this result which is as follows.

THEOREM 2.4. Let $\mathfrak{A}$ be a Banach subalgebra of $C(\Omega)$ which strongly separates the points of $\Omega$. Then $\mathfrak{U}=C(\Omega)$ if the following two conditions are satisfied:

(i) To each pair of points $\omega_{1}, \omega_{2}$ there exist disjoint compact neighborhoods $N_{1}, N_{2}$ of $\omega_{1}, \omega_{2}$ respectively such that $\mathfrak{A} \mid N_{1} \cup N_{2}$ is closed in $C\left(N_{1} \cup N_{2}\right)$

(ii) Each point $\omega$ has a compact neighborhood $N_{\omega}$ such that for each compact set $F \subset N_{\omega}, \mathfrak{U} \mid F$ is closed in $C(F)$.

Proof. We shall apply 2.1. First note that the hypothesis of $\varepsilon$-normality. in 2.1 can be weakened slightly. We need only to insist that for disjoint nonempty compact subsets $F_{1}, F_{2}$ of $\Omega$ there exists an $x \in \mathfrak{U}$ satisfying $\left|x\left(F_{1}\right)\right|<\varepsilon,\left|1-x\left(F_{2}\right)\right|<\varepsilon$. Indeed the argument on pp. 158-9 of [5] shows that (i) implies that for disjoint nonempty compact sets $F_{1}, F_{2}$ of $\Omega$ there exists an $x \in \mathfrak{U}$ for which $x\left(F_{1}\right)=0$, and $x\left(F_{2}\right)=1$.

To complete the proof we observe that for each $\omega$ there exists a compact neighborhood $N_{\omega}$ such that $\mathfrak{A}_{\omega}$ is boundedly normal on $N_{\omega} \sim\{\omega\}$. This follows from condition (ii) by the same argument as in [5, Lemma 3]. To wit: If there exist neighborhoods $U_{i} \subset \bar{U}_{i} \subset U_{i-1}$ of $\omega_{i}$; disjoint compact sets $S_{0, i}, S_{1, i} \subset U_{i-1} \sim \bar{U}_{i}$ such that if $x_{i} \in \mathfrak{A}_{\omega}, x\left(S_{0, i}\right)=0$, 
$x\left(S_{1, i}\right)=1$, then $\left\|x_{i}\right\| \geqq i$, the closed graph theorem implies that it cannot be the case that $\mathfrak{A} \mid E$ is closed in $C(F)$ where $F=\overline{\cup_{i} S_{0, i} \cup S_{1, i} \cup\{\omega\}}$. Therefore $\mathfrak{A} \mid N_{\omega} \sim\{\omega\}=C_{0}(N \sim\{\omega\})$ and consequently $\mathfrak{U} \mid N_{\omega}=C\left(N_{\omega}\right)$. The result now follows from 2.2.

As an application of these techniques we consider the following question:

Let $\Omega$ be compact as before and assume that $\Omega=\Omega_{1} \cup \Omega_{2}, \Omega_{i}$ compact. The example at the beginning of Section 2 shows that if $\mathfrak{U}$ is a Banach subalgebra of $C(\Omega)$ satisfying $\mathfrak{A} \mid \Omega_{i}=C(\Omega), i=1,2$, then it is not necessarily true that $\mathfrak{U} \mid \Omega=C(\Omega)$ even if $\Omega_{1}$ and $\Omega_{2}$ are disjoint. If, however, $\mathfrak{A}$ is normal or even $\varepsilon$-normal, then the disjointness of $\Omega_{1}$ and $\Omega_{2}$ implies $\mathfrak{U} \mid \Omega=C(\Omega)$. This is trivial in the first case and the second is a special case of Theorem 2.2.

If $\Omega_{1} \cap \Omega_{2} \neq \varnothing$, then it is not known whether normality of $\mathfrak{A}$ is sufficient of guarantee that $\mathfrak{A}=C(\Omega)$. In particular let $\mathfrak{A}$ be the algebra of Fourier transforms of $L_{1}(\Gamma)$; and $\Omega_{1}, \Omega_{2}$ be Helson sets in $G$ (cf. Section 1). It is not known whether $\Omega_{1} \cup \Omega_{2}$ is a Helson set.

The following theorem shows that $\mathfrak{U}=C(\Omega)$ if one assumes a certain extension property for ideals.

THEOREM 2.5. Let $\Omega=\Omega_{1} \cup \Omega_{2} ; \Omega_{i}$ compact, and assume $\mathfrak{A}$ is a normal Banach subalgebra of $C(\Omega)$ such that $\mathfrak{A} \mid \Omega_{i}=C\left(\Omega_{i}\right) i=1,2$. For a closed set $F \subset \Omega$, let $F_{i}=\Omega_{i} \cap F$. Let

$$
J_{F^{*}}=\{x \in \mathfrak{U}: x(F)=0\}, \widetilde{J}_{F_{i}}=\left\{x \in C\left(\Omega_{i}\right): x\left(F_{i}\right)=0\right\} .
$$

If $J_{F} \mid \Omega_{i}=\widetilde{J}_{F_{i}}, i=1,2$, for each closed set $F$, then $\mathfrak{A}=C(\Omega)$.

Proof. Let us establish first the following:

Lemma 2.6. For each $\omega \in \Omega$ there exists a neighborhood $N_{\omega}$ of $\omega$ and a constant $M$ such that if $H_{1}, H_{2}$ are disjoint compact subsets of $N_{\omega} \sim\{\omega\}$ and $H_{i} \cap \Omega_{i}=\varnothing$, then there exists $x \in \mathfrak{A}_{\omega}$ satisfying

$$
\begin{aligned}
& x\left(H_{1}\right)=0 \\
& x\left(H_{2}\right)=1 \\
& \|x\| \leqq M .
\end{aligned}
$$

Proof. Note if there exists a neighborhood $N_{\omega} \ni\{\omega\}$ such that $N_{\omega} \cap \Omega_{i}=\varnothing$ for $i=1$ or $i=2$ there is nothing to prove. If the lemma is false, there exists a decreasing sequence of open sets $U_{i} \ni \omega$ such that $U_{i+1} \subset \bar{U}_{i+1} \subset U_{i}$, and disjoint closed sets $H_{i, 1}, H_{i, 2}$ with $H_{i, j} \leqq U_{i} \sim \bar{U}_{i+1}$, such that for each $i H_{i, j} \cap \Omega_{i}=\varnothing, j=1,2$, and if $x_{i} \in \mathfrak{A}_{\omega} ; x_{i}\left(H_{i, 1}\right)=0$, $x_{i}\left(H_{i, 2}\right)=1$ then $\left\|x_{i}\right\| \geqq i$. Let $H=\overline{\cup H_{i, 1} \cup\{\omega\}}$, and $H_{1}=H \cap \Omega_{1}$. Applying the hypothesis together with the closed graph theorem, we 
see that if $\widetilde{x} \in C\left(\Omega_{1}\right), \widetilde{x}\left(H_{1}\right)=0$, and $\|\widetilde{x}\|_{\infty} \leqq 1$, then there is an $x \in J_{H}$ satisfying $x \mid \Omega_{1}=\widetilde{x}$, and $\|x\| \leqq K$. Consequently, since for each $i$ there exists $\widetilde{x}_{i} \in C\left(\Omega_{1}\right)$ satisfying $\widetilde{x}_{i}\left(H_{1}\right)=0, \widetilde{x}_{i}\left(H_{i, 2}\right)=1,\left\|\widetilde{x}_{i}\right\|_{\infty}=1$, it follows that there exists $x_{i} \in J_{H},\left\|x_{i}\right\| \leqq K$, satisfying $x_{i} \mid \Omega_{1}=\widetilde{x}_{i}$. This is a contradiction.

To prove the theorem in view of the normality of $\mathfrak{A}$, it suffices to show that for each $\omega$ there exists a compact neighborhood $N_{\omega} \ni \omega$ such that $\mathfrak{A}_{\omega} \mid N_{\omega}=C_{0}\left(N_{\omega} \sim\{\omega\}\right)$. To prove the latter statement it suffices, by the remark following 1.5 , to show that there exist constants $M_{1}, k, k<1$, such that for each compact set $F \subset N_{\omega} \sim\{\omega\}$ and $f \in C(F)$, $\|f\|_{\infty} \leqq 1$, there exists $\hat{f} \in \mathfrak{A}_{\omega}, \quad\|\hat{f}\| \leqq M_{1}$, and $|(f-\hat{f})(\omega)| \leqq k$ for $\omega \in F$.

Choose $N_{\omega}$ so that 2.6 is satisfied. There exists a constant $N$ depending only on $\Omega_{1}$ and $\Omega_{2}$, so that if $F \subseteq N_{\omega} \sim\{\omega\}$ and $f \in C(F)$, $\|f\|_{\infty} \leqq 1$, then there exist $f_{1}, f_{2} \in \mathfrak{A}_{\omega}$ such that

$$
f_{1}(\omega)=f(\omega), \omega \in F \cap \Omega_{i},\left\|f_{i}\right\| \leqq N, \quad i=1,2 .
$$

Let $M$ be the constant of Lemma 2.6 and $\varepsilon<(2 M)^{-1}$. Let

$$
H_{i}=\left\{\omega \in F:\left|f_{i}(\omega)-f(\omega)\right| \geqq \varepsilon\right\} .
$$

Then $H_{i} \cap \Omega_{i}=\varnothing, i=1,2$, and $H_{1} \cap H_{2}=\varnothing$. We know by Lemma 2.6 there exist functions $x_{i} \in \mathfrak{A}_{\omega}$ satisfying $\left\|x_{i}\right\| \leqq M$ and

$$
\begin{array}{ll}
x_{1}\left(H_{1}\right)=0, & x_{1}\left(H_{2}\right)=1 \\
x_{2}\left(H_{1}\right)=1, & x_{2}\left(H_{2}\right)=0 .
\end{array}
$$

Let $\hat{f}=f_{1} x_{1}+f_{2} x_{2}$. Then $\hat{f} \in \mathfrak{A}_{\omega},\|\hat{f}\| \leqq 2 M N$, and

$$
\begin{aligned}
|(\hat{f}-f)(\omega)| & \leqq\left|\left(f-f_{1}\right)(\omega) x_{1}(\omega)\right|+\left|\left(f-f_{2}\right)(\omega) x_{2}(\omega)\right| \\
& \leqq 2 M \varepsilon, \quad \omega \in F .
\end{aligned}
$$

3. Let us return to the situation when a Banach space $X$ is continuously imbedded by the linear mapping $T$ as an $\varepsilon$-normal family in $C(\Omega), \Omega$ compact Hausdorff. As was remarked in Section 1 some sort of boundedness condition was essential to guarantee that the mapping was onto. We show next that an appropriate condition can be imposed on $\Omega$ which will guarantee that $T$ is locally bounded thus proving that locally $T$ must be onto. The condition we need is due to Gillman and Henriksen [4].

DeFinition 3.1. A compact Hausdorff space $\Omega$ is called an $F$-space if disjoint open $F_{\sigma}$ sets in $\Omega$ have disjoint closures.

The Stone spaces of complete or $\sigma$-complete Boolean algebras as well as their closed sub-spaces have this property. If $C(\Omega)$ is an 
adjoint space, $\Omega$ is extremely disconnected, [7, Theorem 2], and is therefore an $F$-space. There are connected examples such as $\beta\left(R_{+}\right) \sim R_{+}$, where $R_{+}$is the nonnegative reals [4].

REMARK. If $\Omega$ is a compact $F$-space, a subspace $M$ of $C(\Omega)$ which is $\varepsilon$-normal for $\varepsilon<1 / 4$ is necessarily dense in $C(\Omega)$. To prove this it clearly suffices to show that the restriction of $M$ to the carrier of any measure is dense in all continuous functions on the carrier. However, K. Hoffman and (independently) G. Seever [16] have proved that the carrier of any measure on an $F$-space is extremely disconnected. The result now follows from Corollary 1.7. Thus in the next theorems the hypothesis of $\varepsilon$-normality is no gain in generality over density. However it is easier to verify in applications. The result of Hoffman and Seever shows it would suffice to prove these theorems in the case $\Omega$ is extremely disconnected and supports a measure. This observation does not simplify the present proofs.

THEOREM 3.2. Let $\Omega$ be a compact $F$-space, $T$ a continuous imbedding of a Banach space $X$ into $C(\Omega)$ such that $T X$ is \&-normal for some $\varepsilon<1 / 4$. Then there exist finitely many compact sets $S_{1}, \cdots$, $S_{n}$ whose interiors cover $\Omega$ such that

$$
T X \mid S_{i}=C\left(S_{i}\right) \quad i=1, \cdots, n .
$$

Proof. By Corollary 1.6 it suffices to prove that $T X$ is boundedly $\varepsilon$-normal in a neighborhood of each point $\omega_{0}$ of $\Omega$. Since $T X$ is dense in $C(\Omega),(T X)_{\omega_{0}}$ is dense in $C_{0}\left(\Omega \sim\left\{\omega_{0}\right\}\right)$ (see [17]), and by 1.5 it suffices to verify that $(T X)_{\omega_{0}}$ is boundedly $\varepsilon$-normal in a deleted neighborhood of $\omega_{0}$. Suppose, on the contrary, that there exists a sequence of open neighborhoods $V_{i}$ of $\omega_{0}, V_{i+1} \subset \overline{V_{i+1}} \subset V_{i}$, and disjoint compact sets $F_{i, 1}, F_{i, 2} \subset V_{i} \sim \overline{V_{i+1}}$ such that if

$$
\begin{array}{ll}
|(T x-1)(\omega)|<\varepsilon & \omega \in F_{i, 1}, \\
|(T x)(\omega)|<\varepsilon & \omega \in F_{i, 2}, \\
(T x)\left(\omega_{0}\right)=0, &
\end{array}
$$

then $\|x\| \geqq i$. But there exist disjoint open $F_{\sigma}$ sets $G_{i, 1}, G_{i, 2}$ containing $F_{i, 1}, F_{i, 2}$ respectively and contained in $V_{i} \sim \overline{V_{i+1}}$. Now let $G_{1}=\cup_{i} G_{i, 1}$, $G_{2}=\cup_{i} G_{i, 2}$. Then $G_{1}, G_{2}$ are open $F_{\sigma}$ sets with disjoint closures $H_{1}, H_{2}$ respectively. We may assume $\omega_{0} \notin H_{1} \cup H_{2}$. For if $\omega_{0} \in H_{1}$ say, replace $G_{1}$ by $G_{1}^{\prime}=\cup_{i} G_{2 i, 1}$ or $G_{1}^{\prime \prime}=\cup G_{2 i+1,1}$ depending on whether $\omega_{0} \notin \overline{G_{1}^{\prime}}$ or $\omega_{0} \notin \overline{G_{1}^{\prime \prime}}$. Choose $x \in X$ such that $(T x)\left(\omega_{0}\right)=0,|(T x-1)(\omega)|<\varepsilon, \omega \in H_{1}$; $|(T x)(\omega)|<\varepsilon, \omega \in H_{2}$. Then (1) and (2) and (3) are satisfied by $x$, so 
$\|x\|=\infty$. This contradiction completes the proof.

Combining this with Theorem 2.1 we obtain:

TheoRem 3.3. If $\mathfrak{A}$ is an e-normal Banach subalgebra of $C(\Omega)$, $\Omega$ a compact $F$-space, then $\mathfrak{A}=C(\Omega)$.

A known special case of 3.3 is Rudin's interpolation theorem for Sidon sets [15, Theorem 5.74], (Corollary 3.4 below). Recall that a set $E$ in a discrete group $I^{\prime}$ is a Sidon set if for each bounded function $\varphi$ on $E$ there exists a measure $\mu$ on the dual group $G$ satisfying $\hat{\mu}(\gamma)=\varphi(\gamma)$. The restriction of $\hat{\mu}$ to $E$ defines a mapping $T$ of $M(G)$ into $l_{\infty}(E)=C(\beta E)$. Since $\beta E$ is an $F$ space, (c.f. [4] p. 369), an application of 3.3 yields the following.

COROLlaRY 3.4. If $G$ is a compact group with dual group $\Gamma$, then $E \subset \Gamma$ is a Sidon set if for each function $\varphi$ satisfying $\varphi(\gamma)= \pm 1$, $\gamma \in E$; there exists a measure $\mu$ on $G$ satisfying

$$
\sup _{\gamma \in E}|\hat{\mu}(\gamma)-\varphi(\gamma)|<1 \text {. }
$$

A similar statement can be made for interpolating sequences for bounded analytic functions. Following Hoffman [9], call $E \equiv\left\{z_{n}\right\} \cong$ $\{|z|<1\}$ an interpolating sequence if for each bounded function $\varphi$ on $E$ there exists a bounded analytic function on the open unit disc such that

$$
f\left(z_{n}\right)=\varphi\left(z_{n}\right), \quad z_{n} \in E .
$$

Again the restriction of $\varphi$ to $E$ defines a mapping $T$ of the bounded analytic functions into $l_{\infty}=C(\beta N)$. Since $\beta N$ is an $F$ space, Theorem 3.3 yields the following extension of a result of Hayman [8] (see also Hoffman [9, p. 205]).

Corollary 3.5. A sequence $E$ in the open unit disc is an interpolating sequence if for each function $\varphi$ on $E$ such that $\varphi^{2}=\varphi$ there exists a bounded analytic function $f$ satisfying

$$
\sup _{z \in E}|f(z)-\varphi(z)|<\frac{1}{2} .
$$

The intrinsic condition that $E$ be an interpolating sequence, proved by Carleson [2], is that

$$
\prod_{\jmath \neq k}\left|\frac{z_{k}-z_{j}}{1-\bar{z}_{j} z_{k}}\right| \geqq \delta>0 \quad k=1,2, \cdots
$$


To prove Carleson's theorem by the methods discussed here it would suffice to produce for each subset $E_{1} \subset E$ a bounded analytic function vanishing on $E_{1}$ such that $f\left(E \sim E_{1}\right)$ is contained in a compact set not containing the origin and having connected complement. If the sequence $E$ is real this can be accomplished by the Blaschke product having $E_{1}$ as its set of zeroes. Whether the Blaschke products provide this separation in the general case is not known. In view of the known behavior of these functions on the boundary of the unit disc, this is perhaps too much to expect.

CoROllary 3.6. If $\mathfrak{A}$ is a Banach subalgebra of $C(\Omega), \Omega$ a compact $F$ space, then $\mathfrak{H}=C(\Omega)$ if for each pair of points $\omega_{1}, \omega_{2}$ there exists an $x \in \mathfrak{A}$ which vanishes in a neighborhood of $\omega_{1}$ and equals one in a neighborhood of $\omega_{2}$.

Proof. As in the proof of 2.4. the condition implies that for each pair of disjoint nonempty compact subsets $F_{1}, F_{2}$ there exists an $x \in \mathfrak{A}$ satisfying $x\left(F_{1}\right)=0 ; x\left(F_{2}\right)=1$. By 3.3 for each $\omega \in \Omega$, there is a compact neighborhood $N_{\omega}$ of $\omega$ such that $\mathfrak{A} \mid N_{\omega}=C\left(N_{\omega}\right)$. An application of 2.1 completes the proof.

One should not expect that the condition of 3.6 can be substantially weakened. For recently Hoffman and Ramsey [10] have shown that if one assumes the continuum hypothesis then separating closed subalgebras of $l_{\infty}$ exist in great abundance.

4. This final section contains some results and examples concerned with the problem of extending Theorem 3.2. In an earlier version of the manuscript we had conjectured that if $\Omega$ is a compact $F$-space and $T: X \rightarrow C(\Omega)$ is a continuous linear map of a Banach space with dense range, then $T X=C(\Omega)$. We are grateful to J. Lindenstrauss for the following elegant counterexample to this conjecture:

There exists a continuous linear map $\Phi$ from $L_{\infty}(0,1)$ onto $l_{2}$, since $L_{1}(0,1)$ contains a subspace isomorphic to $l_{2}$ (e.g. the subspace generated by the Rademacher functions). Let $\left\{e_{n}\right\}, n=1,2, \cdots$, be an orthonormal basis in $l_{2}$ and $E_{n}=s p\left\{e_{i}, \cdots, e_{n}\right\}$. Let $X_{n}=\Phi^{-1}\left(E_{n}\right)$ and let $X$ be the Banach space of all sequences $x=\left\{x_{n}\right\}$, with $x_{n} \in X_{n}$ and $\|x\|=\sup _{n}\left\|x_{n}\right\|<\infty$. Let $T: X \rightarrow L_{\infty}(0,1)$ be defined by

$$
T x=T\left(\left\{x_{n}\right\}\right)=\sum_{n=1}^{\infty} \frac{x_{n}}{n !} .
$$

Then $L_{\infty}(0,1)$ is a space $C(\Omega)$, where $\Omega$ is stonian, and $T X$ is dense in $L_{\infty}(0,1)$ as it contains $\cup_{n=1}^{\infty} X_{n}$. Also $T X$ is not the whole of $L_{\infty}(0,1)$, 
since if there were an $x=\left\{x_{n}\right\}$ in $X$ such that

$$
\Phi(T x)=\sum_{n=1}^{\infty} \frac{\Phi\left(x_{n}\right)}{n !}=\sum_{n=1}^{\infty} \frac{e_{n}}{n}
$$

then one must have

$$
\left\|\sum_{n=N+1}^{\infty} \frac{\Phi\left(x_{n}\right)}{n !}\right\| \geqq\left\|\sum_{n=N+1}^{\infty} \frac{e_{n}}{n}\right\|
$$

in $l_{2}$, for $N=1,2, \cdots$, as $\Phi\left(x_{n}\right) \in E_{n}$. Since $\sup _{n}\left\|\Phi\left(x_{n}\right)\right\|<\infty$, we obtain the desired contradiction.

We know of two cases one can conclude that $T X=C(\Omega)$ under additional hypotheses. G. Seever [16] has proved that $T X=C(\Omega)$ if $T X$ is normal on $\Omega$. We shall give a new proof.

Theorem 4.1. (Seever) Let $\Omega$ be a compact $F$-space and $T: X \rightarrow$ $C(\Omega)$ be a continuous linear map of a Banach space such that $T X$ is normal on $\Omega$. Then $T X=C(\Omega)$.

By earlier remarks one can suppose that $\Omega$ is totally disconnected. Seever proves that if $T X$ is normal on $\Omega, T^{*}: C(\Omega)^{*} \rightarrow X^{*}$ has a closed range. This fact rests on a uniform boundedness theorem for measures on totally disconnected $F$-spaces which is derived from a theorem of R.S. Phillips [15, p. 525] on convergence of finitely additive measures on the subsets of the integers. Since $T X$ is dense in $C(\Omega)$ and has a closed range it follows that $T X=C(\Omega)$. The proof we shall give also relies on Seever's reduction to the case that $\Omega$ is totally disconnected. The necessary element of uniformity is supplied by the following lemma.

Lemma 4.2. Let $\Omega$ be a totally disconnected $F$-space and $T X$ be normal on $\Omega$. For each point $\omega_{0} \in \Omega$ there exists an open and closed neighborhood $V$ and constant $K$ such that if $E_{1}$ and $E_{2}$ are any disjoint compact and open subsets of $V \sim\left\{\omega_{0}\right\}$, one can find $x \in X$ such that

$$
\begin{aligned}
& (T x)\left(E_{1}\right)=1, \quad(T x)\left(E_{2}\right)=0, \quad(T x)\left(V^{\prime}\right)=0 \\
& (T x)\left(\omega_{0}\right)=0, \quad\|x\| \leqq K
\end{aligned}
$$

Proof. Suppose the lemma is false. If $W_{1}$ is an open and closed neighborhood of $\omega_{0}$, there exist disjoint compact and open subsets $E_{11}$, $E_{12}$ of $W_{1} \sim\left\{\omega_{0}\right\}$ such that if $x \in X$ and $(T x)\left(E_{11}\right)=1,(T x)\left(E_{12} \cup W_{1}^{\prime}\right)=0$, $(T x)\left(\omega_{0}\right)=0$, then $\|x\| \geqq 2$. Let $k_{E}$ denote the characteristic function of a set $E$. Using the assumption of normality, select $x_{1} \in X$ such 
that $T x_{1}=k_{E_{11}}$, and let $W_{2}$ be an open and closed neighborhood of $\omega_{0}$ with $W_{2} \subseteq W_{1} \sim\left(E_{11} \cup E_{12}\right)$. Proceeding inductively we construct decreasing open and closed neighborhoods $W_{n}$ of $\omega_{0}$ and disjoint open and closed subsets $E_{n 1}, E_{n 2}$ of $W_{n} \sim W_{n+1}$ and elements $x_{n} \in X$ such that if

$$
(T x)\left(E_{n 1}\right)=1,(T x)\left(E_{n 2} \cup W_{1}^{\prime}\right)=0,(T x)\left(\omega_{0}\right)=0,
$$

then

$$
\|x\| \geqq 2^{n}+\sum_{i=1}^{n-1}\left\|x_{i}\right\|
$$

Note that

$$
E_{11} \subseteq E_{11} \cup E_{21} \subseteq E_{11} \cup E_{21} \cup E_{31} \subseteq \cdots \subseteq W_{3} \cup E_{11} \cup E_{21} \subseteq W_{2} \cup E_{11} \subseteq W_{1} .
$$

Since $\Omega$ is an $F$-space, there exists an open and closed set $F_{0}$ such that

$$
\bigcup_{i=1}^{n} E_{i 1} \subseteq F_{0} \subseteq W_{n} \cup \bigcup_{i=1}^{n-1} E_{i 1}, \quad n=1,2, \cdots
$$

Then

$$
k_{F_{0}}(\omega)=\sum_{n=1}^{\infty}\left(T x_{n}\right)(\omega)=\sum_{n=1}^{\infty} k_{E_{n 1}}(\omega)
$$

for $\omega \in \bigcup_{i=1}^{\infty} W_{1}^{\prime}$. By dropping, if necessary, to the subsequences for $n$ even or $n$ odd we can suppose $\omega_{0} \notin F_{0}$. Choose $x_{0}$ with $T x_{0}=k_{F_{0}}$ and define $z_{n}=x_{0}-\sum_{i=1}^{n-1} x_{i}$. Then $T z_{n}$ satisfies (\#), so $\left\|z_{n}\right\| \geqq 2^{n}+\sum_{i=1}^{n-1}\left\|x_{i}\right\|$. This implies $\left\|x_{0}\right\| \geqq 2^{n}, n=1,2, \cdots$, which is the required contradiction. It follows now by the arguments of Theorem 1.5 that $T X$ contains all continuous functions which vanish outside of $V$. A covering argument completes the proof of Theorem 4.1.

One also has $T X=C(\Omega)$ is the special case that $X$ is a conjugate space, $C(\Omega)$ is $l_{\infty}$ and $T$ is weak star continuous. This theorem is essentially an unpublished result of Beurling.

THEOREM 4.3. (Beurling) Let $S$ be an arbitrary set and $X$ be a Banach space. If $T: l_{1}(S) \rightarrow X$ is linear and continuous and $T^{*}: X^{*} \rightarrow$ $l_{\infty}(S)$ has dense range then $T^{*} X^{*}=l_{\infty}(S)$.

Proof. The density of $T^{*} X^{*}$ shows $T$ is one to one. We prove that $T^{-1}$ is bounded, so $T$ and hence $T^{*}$ has a closed range. If $T^{-1}$ is not bounded we can find a sequence $\left\{\xi_{n}\right\}$ of elements of $l_{1}(S)$ of norm one such that $T \xi_{n}$ converges to zero. Thus

$$
\left(T^{*} x^{*}\right)\left(\xi_{n}\right)=\sum_{S} \xi_{n}(s)\left(T^{*} x^{*}\right)(s) \rightarrow 0, \quad x^{*} \in X^{*},
$$


as $n \rightarrow \infty$. Since $T^{*} X^{*}$ is dense in $l_{\infty}(S),\left\{\xi_{n}\right\}$ converges weakly to zero. But then $\left\{\xi_{n}\right\}$ converges strongly to zero ([3], p. 295), giving the desired contradiction.

In a seminar in 1960 Beurling gave a proof (unpublished) of the following theorem equivalent to the result of Rudin (Corollary 3.4) for the case of the circle group.

THEOREM 4.4. Let $\left\{n_{j}\right\}$ be a sequence of integers, and suppose that for each $\varepsilon>0$ and $\left\{\alpha_{i}\right\}$ in $l_{\infty}$ there is a measure $\mu_{\varepsilon}$ on the circle $\Gamma$ such that

$$
\left|\hat{\mu}_{\varepsilon}\left(n_{j}\right)-\alpha_{j}\right|<\varepsilon, \quad j=1,2, \cdots .
$$

Then for each $\left\{\alpha_{j}\right\} \in l_{\infty}$ there exists a measure $\mu$ such that $\hat{\mu}\left(n_{j}\right)=\alpha_{j}$, $j=1,2, \cdots$.

Helson observed that the essential argument of Beurling's proof gave a proof of 4.3. On the other hand, to prove Theorem 4.4 from 4.3 one defines $T\left(\left\{\xi_{n}\right\}\right)=\sum_{j=1}^{\infty} \xi_{j} e^{i j^{t}}$. Then $T: l_{1} \rightarrow C(\Gamma), \Gamma$ the unit circle, and $T^{*}\left(C(\Gamma)^{*}\right)$ is dense in $l_{\infty}$, so the restrictions of the Fourier transforms of measures must yield all bounded sequences. One may also base a proof of Corollary 3.5 on Theorem 4.2.

Finally we give an example of a compact Hausdorff space $\Gamma$, which is not an $F$-space, and a continuous map $T: X \rightarrow C(\Gamma)$ such that $T X$ is dense and normal on $\Gamma$ and enjoys the local matching property of Theorem 3.2, yet for which $T X \neq C(\Gamma)$. This example is due to Y. Katznelson. We denote the $n$-th Fourier coefficient of a continuous function $x$ on the unit circle $\Gamma$ by $\hat{x}_{n}$. Let $X$ be the subspace of $C(\Gamma)$ for which $\sum_{n=-\infty}^{\infty}\left|\hat{x}_{2 n+1}\right|<\infty$. We may write $X=Y \oplus Z$, where

$$
\begin{array}{ll}
Y=\left\{y \in C(\Gamma): \widehat{y}_{2 n+1}=0,\right. & n=0, \pm 1, \pm 2, \cdots\} \\
Z=\left\{z \in C(\Gamma): \widehat{z}_{2 n}=0, \quad n=0, \pm 1, \pm 2, \cdots, \sum_{n=-\infty}^{\infty}\left|\widehat{z}_{2 n+1}\right|<\infty\right\} .
\end{array}
$$

For $x=y+z$ define

$$
\|x\|=\sup _{t \in \Gamma}|y(t)|+\sum_{n=-\infty}^{\infty}\left|\widehat{z}_{2 n+1}\right| .
$$

Then $T X$ is a dense and normal subspace of $C(\Gamma)$ complete in this norm. If $f$ is any continuous function defined on an are of length less than $\pi$, we may construct a continuous function $y$ of period $\pi$ and hence in $Y$, which extends $f$. Thus if $T$ is the injection map of $X$ into $C(\Gamma), \Gamma$ is covered by closed arcs $S_{1}, S_{2}, S_{3}$ such that $T X \mid S_{i}=C\left(S_{i}\right)$ but $T X \neq C(\Gamma)$. 


\section{BIBLIOGRAPHY}

1. W. G. Bade and P. C. Curtis, Jr., The Wedderburn decomposition of commutative Banach algebras, Amer. J. Math. 82 (1960), 851-866.

2. L. Carleson, An interpolation problem for bounded analytic functions, Amer. J. Math. 80 (1958), 921-930.

3. N. Dunford, and J. Schwartz, Linear Operators, Part I: General theory, New York, 1958.

4. L. Gillman, and M. Henriksen, Rings of continuous functions in which every finitely generated ideal is principal, Trans. Amer. Math. Soc. 82 (1956), 366-391.

5. I. Glicksberg, Function algebras with closed restrictions. Proc. Amer. Soc. 14 (1963), $155-161$.

6. E. A. Gorin, A property of the ring of all continuous functions on a bicompactum, Doklady Akad. Nauk. SSSR (N.S.) 142 (1962), 781-784 (Soviet Math. 3 (1962), 159-162). 7. A. Grothendieck, Une caractérisation des espaces $L^{1}$, Canadian J. Math. 7 (1955), $552-561$.

8. W. Hayman, Interpolation by bounded functions, Ann. de l'Institute Fourier 13 (1958), 277-290.

9. K. Hoffman, Banach Spaces of Analytic Functions, Englewood Cliffs, 1962.

10. K. Hoffman, and A. Ramsey, Algebras of bounded seguences, Pacific J. Math. 15 (1965), 1239-1248.

11. Y. Katznelson, A characterization of the algebra of all continuous functions on a compact Hausdorff space, Bull. Amer. Math. Soc. 66 (1960), 313-315.

12. Characterization of $C(\mathfrak{M})$, Technical Note No. 25, Air Force Office of Scientific Research, Hebrew University, 1962.

13. R. McKissick, A non trivial sup norm algebra, Bull. Amer. Math. Soc. 69 (1963), 391-395.

14. R. S. Phillips, On linear transformations, Trans. Amer. Math. Soc. 48 (1940), 516-541.

15. W. Rudin, Fourier Analysis on groups, New York, 1962.

16. G. Seever, Measures on F-spaces, Thesis, University of California, Berkeley, 1963.

17. H. Yamabe, On an extension of Helly's theorem, Osaka Math. J. 2 (1950), 15-17.

Received April 20, 1964 and in revised form July 6, 1964. This work was supported by the Air Force of Scientific Research and the National Science Foundation.

University of California, Berkeley

UNiversity of CALIFornia, Los ANGELes 



\section{PACIFIC JOURNAL OF MATHEMATICS}

\section{EDITORS}

\author{
H. SAMELSON, \\ Stanford University \\ Stanford, California \\ R. M. BLUMENTHAL \\ University of Washington \\ Seattle, Washington 98105
}

\author{
*J. DugundJI \\ University of Southern California \\ Los Angeles, California 90007 \\ RICHARD ARENS \\ University of California \\ Los Angeles, California 90024
}

\section{ASSOCIATE EDITORS}
E. F. BECKENBACH
B. H. NeUmanN
F. WOLF
K. YOSIDA

\section{SUPPORTING INSTITUTIONS}

\author{
UNIVERSITY OF BRITISH COLUMBIA \\ CALIFORNIA INSTITUTE OF TECHNOLOGY \\ UNIVERSITY OF CALIFORNIA \\ MONTANA STATE UNIVERSITY \\ UNIVERSITY OF NEVADA \\ NEW MEXICO STATE UNIVERSITY \\ OREGON STATE UNIVERSITY \\ UNIVERSITY OF OREGON \\ OSAKA UNIVERSITY \\ UNIVERSITY OF SOUTHERN CALIFORNIA
}

\author{
STANFORD UNIVERSITY \\ UNIVERSITY OF TOKYO \\ UNIVERSITY OF UTAH \\ WASHINGTON STATE UNIVERSITY \\ UNIVERSITY OF WASHINGTON \\ * * * \\ AMERICAN MATHEMATICAL SOCIETY \\ CHEVRON RESEARCH CORPORATION \\ TRW SYSTEMS \\ NAVAL ORDNANCE TEST STATION
}

Mathematical papers intended for publication in the Pacific Journal of Mathematics should be typewritten (double spaced). The first paragraph or two must be capable of being used separately as a synopsis of the entire paper. It should not contain references to the bibliography. Manuscripts may be sent to any one of the four editors. All other communications to the editors should be addressed to the managing editor, Richard Arens at the University of California, Los Angeles, California 90024 .

50 reprints per author of each article are furnished free of charge; additional copies may be obtained at cost in multiples of 50 .

The Pacific Journal of Mathematics is published monthly. Effective with Volume 16 the price per volume (3 numbers) is $\$ 8.00$; single issues, $\$ 3.00$. Special price for current issues to individual faculty members of supporting institutions and to individual members of the American Mathematical Society: $\$ 4.00$ per volume; single issues $\$ 1.50$. Back numbers are available.

Subscriptions, orders for back numbers, and changes of address should be sent to Pacific Journal of Mathematics, 103 Highland Boulevard, Berkeley 8, California.

Printed at Kokusai Bunken Insatsusha (International Academic Printing Co., Ltd.), No. 6, 2-chome, Fujimi-cho, Chiyoda-ku, Tokyo, Japan.

PUBLISHED BY PACIFIC JOURNAL OF MATHEMATICS, A NON-PROFIT CORPORATION

The Supporting Institutions listed above contribute to the cost of publication of this Journal, but they are not owners or publishers and have no responsibility for its content or policies.

* Paul A. White, Acting Editor until J. Dugundji returns. 


\section{Pacific Journal of Mathematics}

\section{Vol. 18, No. 3 \\ May, 1966}

William George Bade and Philip C. Curtis, Jr., Embedding theorems for commutative Banach algebras .......................... 391

Wilfred Eaton Barnes, On the $\Gamma$-rings of Nobusawa ................. 411

J. D. Brooks, Second order dissipative operators ................ 423

Selwyn Ross Caradus, Operators with finite ascent and descent ........ 437

Earl A. Coddington and Anton Zettl, Hermitian and anti-hermitian properties of Green's matrices .......................... 451

Robert Arnold Di Paola, On sets represented by the same formula in distinct consistent axiomatizable Rosser theories ................... 455

Mary Rodriguez Embry, Conditions implying normality in Hilbert space ...........................................

Garth Ian Gaudry, Quasimeasures and operators commuting with convolution ................................... 461

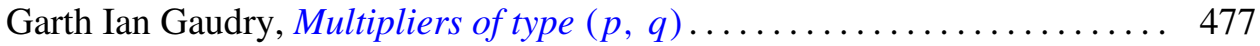

Ernest Lyle Griffin, Jr., Everywhere defined linear transformations affiliated with rings of operators .................................. 489

Philip Hartman, On the bounded slope condition ................ 495

David Wilson Henderson, Relative general position ................ 513

William Branham Jones, Duality and types of completeness in locally

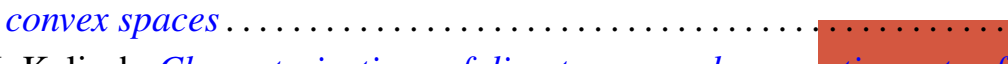

G. K. Kalisch, Characterizations of direct sums and commuting sets of Volterra operators....

Ottmar Loos, Über eine Beziehung zwischen Malcev-Algebren und Lietripelsystemen

Ronson Joseph Warne, A class of bisimple inverse semigroups . . . 